\title{
Electrically tunable filter based on plasmonic phase retarder and liquid crystals
}

\author{
Luc Driencourt ${ }^{\mathrm{a}}$, François Federspiel $^{\mathrm{b}}$, Dimitrios Kazazis $^{\mathrm{c}}$, Li-Ting Tseng ${ }^{\mathrm{c}}$, Richard Frantz ${ }^{\mathrm{b}}$, \\ Yasin Ekinci ${ }^{\mathrm{c}}$, Rolando Ferrini ${ }^{\mathrm{a}}$, and Benjamin Gallinet ${ }^{\mathrm{a}}$ \\ ${ }^{a}$ CSEM Muttenz, Tramstrasse 99, CH-4132 Muttenz \\ ${ }^{\mathrm{b}}$ Rolic Technologies Ltd., Allschwil, Switzerland \\ cPaul Scherrer Institut (PSI), Villigen, Switzerland
}

\begin{abstract}
An electrically tunable filter based on a plasmonic phase retarder and liquid crystal cells is reported. The plasmonic phase retarder consists of a periodic array of deep-subwavelength metallic nanostructures. A first entrance polarizer prepares the incident light in a polarization state oriented at $45^{\circ}$ from the nanowires orientation. A strong phase retardation between TM and TE polarizations is induced by the plasmon resonances. A polarization analyzer based on liquid crystal cells allows to project the transmitted light onto a polarization state whose orientation depends on the applied voltage. Using this approach, a range of $8 \mathrm{~V}$ is enough to span more than $70 \%$ of the area covered by standard RGB filters in CIE color coordinates with a single filter, including yellow, orange, red, magenta, purple, blue, cyan and green as well as different tones of white. In order to ensure the applicability to large area production, UV nanoimprint lithography (UV-NIL) and thin film coatings have been used to fabricate the plasmonic phase retarder. The evaporation is performed with an angle, so that a self-shadowing effects prevents full coverage of the surface. The resulting structure consists in a periodic array of silver nanowires. Multiple interfering resonances are observed so that the nominal transmission can reach levels above $70 \%$. The construction of the colors transmitted by the tunable filter is modeled and validated through a series of optical characterization of the individual elements.
\end{abstract}

Keywords: Plasmonic colors, liquid crystals, tunable filters, nanoimprint lithography, color display, multispectral imaging

\section{INTRODUCTION}

Surface plasmon resonances in metallic nanostructures enable the confinement and manipulation of the electromagnetic field well below the diffraction limit, thus opening new paradigms for optical devices. In addition, subwavelength resonators are able to act on the phase of the light, making them essential building blocks for metasurfaces. This project aims to the development of a colored optical retarder and its integration in optoelectronic devices. This is achieved by design and fabrication of plasmonic nanostructures showing a strong and spectrally narrow birefringent effect. This color effect which can be actively controlled by e.g. liquid crystals. We also show that birefringent-induced colors of the liquid crystal cell combined with the plasmonic phase retarder yield to a green color for high voltages, which is highly desirable for e.g. camera or display filters.

The basic working principle of the device is shown in Fig. 1a. A plasmonic phase retarder can generate different colors in transmission when placed between crossed polarizers and the output polarization is rotated. ${ }^{1,2}$ The plasmonic phase retarder consists of a periodic array of deep-subwavelength metallic nanowires (Fig. 1c). The light polarized across the nanowires (TM) excites a plasmon resonance. A first entrance polarizer prepares the incident light in a polarization state oriented at $45^{\circ}$ from the nanowires orientation. A phase retardation between TM and TE polarization yields an elliptically polarized light in transmission. A polarization analyzer based on liquid crystal cells allows to project the transmitted light onto a polarization state whose orientation depends on the applied voltage. Interferences between the different birefringent contributions lead to a color effect in transmission.

Further author information: (Send correspondence to B.G.) E-mail: benjamin.gallinet@csem.ch

High Contrast Metastructures IX, edited by Connie J. Chang-Hasnain, Andrei Faraon, Weimin Zhou, Proc. of SPIE Vol. 11290, 112901A - (C) 2020 SPIE · CCC code: 0277-786X/20/\$21 · doi: 10.1117/12.2543569 


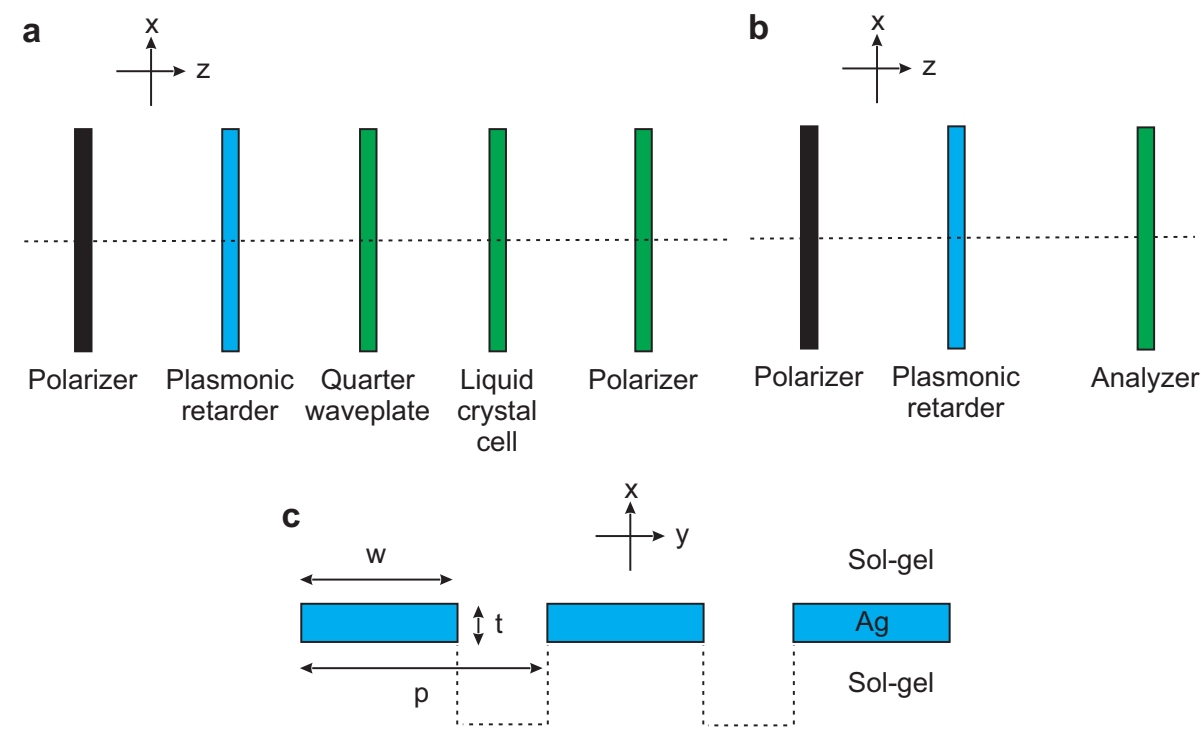

Figure 1. (a) Elements of the filtering system with an entrance polarizer oriented at $45^{\circ}$ from the $x$ and $y$ axis, the plasmonic retarder and a voltage-controlled polarization analyzer (VCPA) comprising a quarter waveplate, a liquid crystal cell (LCC) with main retardation axes rotated by $45^{\circ}$ from the main retardation axes of the waveplate, and a polarizer with fixed orientation. The effective orientation of the analyzer is rotated when a voltage is applied to the LCC. TM and TE polarizations are oriented along $x$ and $y$ axis respectively. The plasmonic nanostructures are aligned with the $y$ axis. (b) Reference filtering system with an analyzing polarizer in place of the VCPA in order to isolate the color contribution of the plasmonic retarder. (c) Schematic cross section of the plasmonic retarder consisting effectively in an array of silver nanowire embedded in a sol-gel matrix.

Using this approach, a range of $8 \mathrm{~V}$ is enough to span more than $70 \%$ of the area covered by standard RGB filters in CIE color coordinates with a single filter, including yellow, orange, red, magenta, purple, blue, cyan and green as well as different tones of white. ${ }^{3}$ In this proceeding, we focus the discussion on the modeling of the system and the optimization of the nanostructure parameters in order to obtain the larget color gamut. The model is validated against experimental measurements.

\section{NANOSTRUCTURE OPTIMIZATION}

The electro-optical response of the system of Fig. 1a is modeled using Jones matrices. The entrance polarizer is placed at $45^{\circ}$ from the polarization axis of the plasmonic nanostructure. The field transmitted by the plasmonic nanostructure is generally described by a Jones vector $T_{\text {in }}$ including a TM-polarized amplitude coefficient $T_{\mathrm{TM}}$, a TE-polarized amplitude coefficient $T_{\mathrm{TE}}$ and a phase delay $\Delta \phi$ between the two polarizations. These responses depend on the wavelength, which is kept implicit in the following for clarity. The system also comprises a quarter-wavelength plate, a liquid crystal cell with fast and slow axis rotated by $45^{\circ}$ from the axis of the quarter-wavelength plate, and a polarizer with fixed orientation, represented by their respective matrices $\Lambda_{4}, L$ and $P_{\text {out }}$. The amplitude of the field transmitted through the system is therefore:

$$
\begin{aligned}
T_{\mathrm{out}} & =P_{\mathrm{out}} \cdot L \cdot \Lambda_{4} \cdot T_{\mathrm{in}} \\
& =\left(\begin{array}{ll}
1 & 0 \\
0 & 0
\end{array}\right) \cdot\left(\begin{array}{cc}
\cos \left(\delta_{V} / 2\right) & -i \sin \left(\delta_{V} / 2\right) \\
-i \sin \left(\delta_{V} / 2\right) & \cos \left(\delta_{V} / 2\right)
\end{array}\right) \cdot\left(\begin{array}{cc}
e^{-i \pi / 4} & 0 \\
0 & e^{i \pi / 4}
\end{array}\right) \cdot\left(\begin{array}{c}
\frac{T_{\mathrm{TM}}}{\sqrt{2}} e^{i \Delta \phi} \\
\frac{T_{\mathrm{TE}}}{\sqrt{2}}
\end{array}\right) \\
& =\frac{1}{\sqrt{2}}\left(T_{\mathrm{TM}} e^{i \Delta \phi} \cos \left(\delta_{V} / 2\right)+T_{\mathrm{TE}} \sin \left(\delta_{V} / 2\right)\right)
\end{aligned}
$$


where $\delta_{V}$ is the phase delay induced by the voltage-dependent birefringence $\delta_{V} n(V)$ in the liquid crystal material of thickness $t$ and can be modeled by:

$$
\delta_{V}=\frac{2 \pi \delta_{V} n(V) t}{\lambda}
$$

The intensity of the transmitted field can be simplified to:

$$
\left|T_{\text {out }}\right|^{2}=\frac{\left[T_{\mathrm{TM}} \cos \left(\delta_{V} / 2\right)\right]^{2}}{2}+\frac{\left[T_{\mathrm{TE}} \sin \left(\delta_{V} / 2\right)\right]^{2}}{2}+T_{\mathrm{TM}} T_{\mathrm{TE}} \cos (\Delta \phi) \cos \left(\delta_{V} / 2\right) \sin \left(\delta_{V} / 2\right)
$$

In the case of a linearly polarized input light $T_{\text {in }}=(\cos \theta, \sin \theta)^{\mathrm{T}}$, the system simplifies to a polarization analyzer whose axis depends on the applied voltage: $\left|T_{\text {out }}\right|^{2}=\cos ^{2}\left(\theta-\delta_{V} / 2\right)$.

Similar Jones-matrix calculation through a system between a polarizer at $45^{\circ}$ and a rotating analyzer with orientation $\theta$ (Fig. 1b) yields for the transmitted amplitude: ${ }^{1,2}$

$$
T_{\text {out }}=\left(\begin{array}{cc}
\cos ^{2} \theta & -\sin \theta \cos \theta \\
-\sin \theta \cos \theta & \sin ^{2} \theta
\end{array}\right) \cdot\left(\begin{array}{cc}
\sqrt{T_{\mathrm{TM}}} e^{i \Delta \phi} & 0 \\
0 & \sqrt{T_{\mathrm{TE}}}
\end{array}\right)\left(\begin{array}{l}
1 / \sqrt{2} \\
1 / \sqrt{2}
\end{array}\right)
$$

and the transmitted intensity:

$$
\left|T_{\text {out }}\right|^{2}=T_{\mathrm{TM}} \cos ^{2} \theta / 2+T_{\mathrm{TE}} \sin ^{2} \theta / 2+T_{\mathrm{TM}} T_{\mathrm{TE}} \cos (\Delta \phi) \cos (\theta) \sin (\theta) .
$$

where $\Delta \phi$ is the phase retardation and $T_{\mathrm{TM}}$ and $T_{\mathrm{TE}}$ are the measured transmittance coefficients when the $\theta=\pi / 2$ and 0 , respectively. The related spectra and CIE color plot are given in Fig. 2. The numerical calculations of the amplitude and phase transmitted by the plasmonic retarder are performed with rigorous coupled wave analysis, which is particularly suited for resonant grating calculations. ${ }^{4}$ The spectra and resulting colors as a function of the orientation of the polarization analyzer are calculated using Eq. (7). The silver permittivity is taken from Johnson and Christy. ${ }^{5}$ In order to account for fabrication imperfections, the imaginary part of the silver permittivity has been multiplied by 2 .

The plasmonic retarder is fabricated by UV nanoimprint lithography and oblique evaporation of silver, which is an upscalable approach to generating isolated nanowire arrays with large transmission. ${ }^{6,7}$ The structure is then encapsulated with sol-gel material. The imprint master consists of a binary grating and is fabricated by electron beam lithography. Using such a manufacturing approach, the design parameters are therefore restricted to the periodicity, the silver thickness and the wire width (related to the underlying shape of the grating master). The minimal feature size should also take into account limitations of the manufacturing process. For periodicities of $180 \mathrm{~nm}$ and above, Wood-Rayleigh anomalies are observed in the visible range for the range of tilt angles investigated. The grating periodicity is chosen as $160 \mathrm{~nm}$ in order to minimize the dependency of the transmission spectrum in the tilt angle, as shown in Fig. 2a. In a second step (Fig. 2b), the optimal nanowire width is chosen as the one which maximizes the color gamut, which is around $105 \mathrm{~nm}$.

The third step consists in optimizing the silver layer thickness, as shown in Fig. 2c. The color gamut is the broadest for a thickness of $20 \mathrm{~nm}$, and a transmission up to 0.7 is observed in the blue range. For higher silver thicknesses, the transmission is significantly lower and the gamut reduced.

\section{VALIDATION OF ELECTRO-OPTICAL MODEL}

In Fig. 4, the model of Eq. (7) is used to reconstruct the transmission of the system consisting of the plasmonic retarder between polarizers (setup of Fig. 1b). Ellipsometric data for the phase retardation $\Delta \phi$ and spectrometric data for the amplitudes $T_{\mathrm{TM}}$ and $T_{\mathrm{TE}}$ are considered. Overall, a good agreement with measurements is observed. ${ }^{3}$ In particular, the $\cos (\Delta \phi)$ spectrum shows how the phase retardation contributes to the orange and red colors for angles around pi/4. Comparing the experimental data with the modeling of Fig. 2 and Fig. 3, a good agreement in spectrum and color is obtained for the different orientations of the analyzing polarizer. Transmission amplitudes are deviating owing to fabrication imperfections compared to the design. Overall, a broad range of colors can be obtained with the plasmonic retarder between polarizers, but it can be further enhanced with the VCPA. 

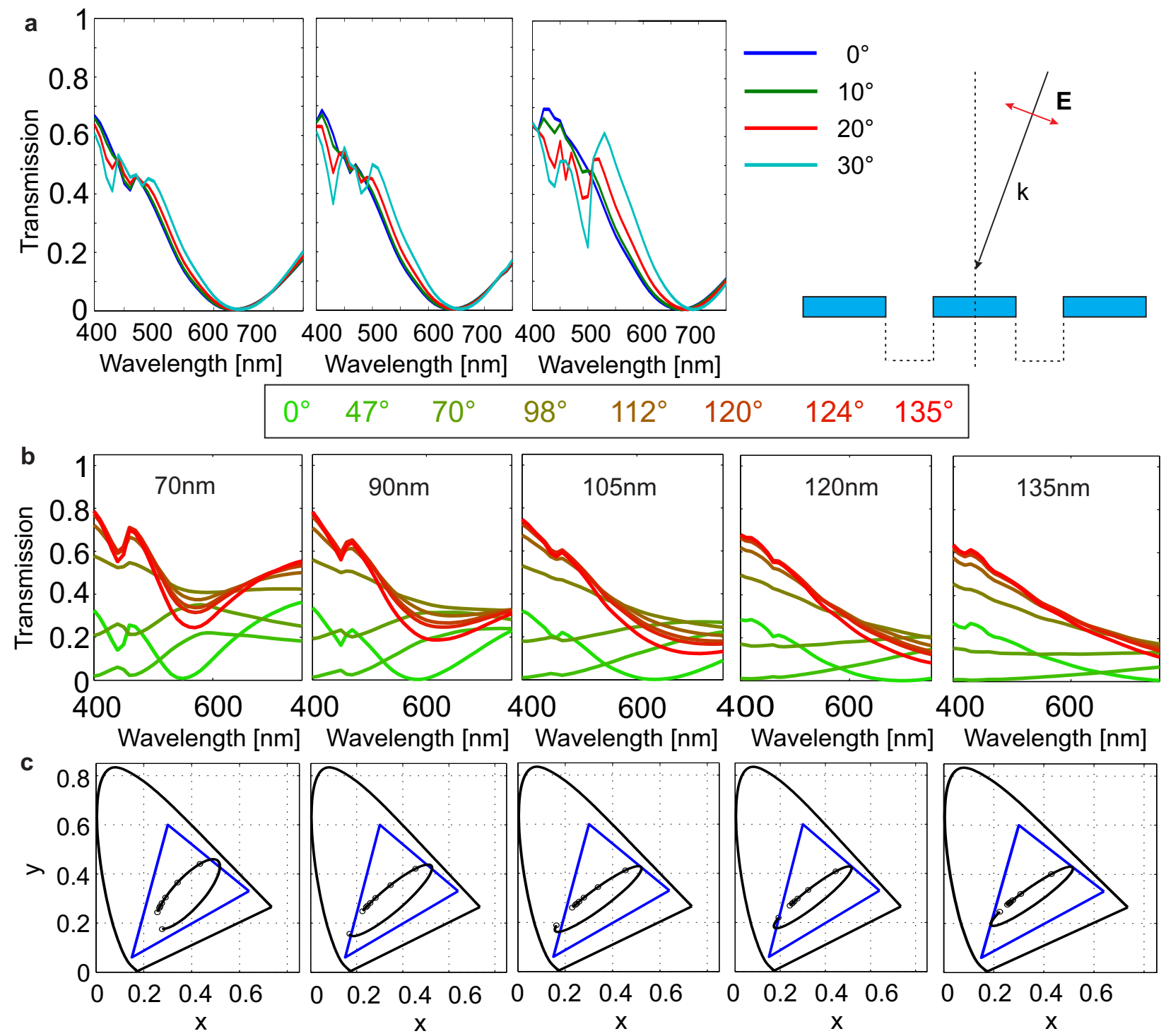

Figure 2. (a) Polarized transmission for periods $p=160 \mathrm{~nm}, 180 \mathrm{~nm}$ and $200 \mathrm{~nm}$ as a function of incidence wavelength and angle, defined from the normal of the plasmonic retarder plane. The electric field is TM polarized, as shown on the right inset. The silver thickness $t$ is $20 \mathrm{~nm}$ and the size of the silver wires $w=0.7 p$. (b) Polarized transmittance as a function of wavelength and analyzer orientation for different nanowire sizes (setup of Fig. 1b). The period is 160nm and the silver thickness is $20 \mathrm{~nm}$. (c) Corresponding CIE color plot.

In Fig. 5, the model of Eq. (5) is used to reconstruct the transmission of the system with and without the plasmonic nanostructure. The coefficients $\cos \left(\delta_{V} / 2\right)$ and $\sin \left(\delta_{V} / 2\right)$ are extracted from transmission measurements of the VCPA combined with a polarizer. Overall, a good agreement with measurements of the full system is observed. ${ }^{3}$ Comparing Fig. 5a and Fig. 5b, a reduction of the FWHM can be observed for high voltages thanks to the plasmonic nanostructure, leading to an increase of the green color saturation.

\section{CONCLUSION}

A broad variety of colors can be spanned upon application of a voltage in the range between $2 \mathrm{~V}$ and $6.5 \mathrm{~V}$ (Fig. 5c). In particular, red is obtained from the plasmonic structure, blue from the LCC and green from the combination of cyan and yellow from the plasmonic structure and the LLC, respectively. Other colors such 

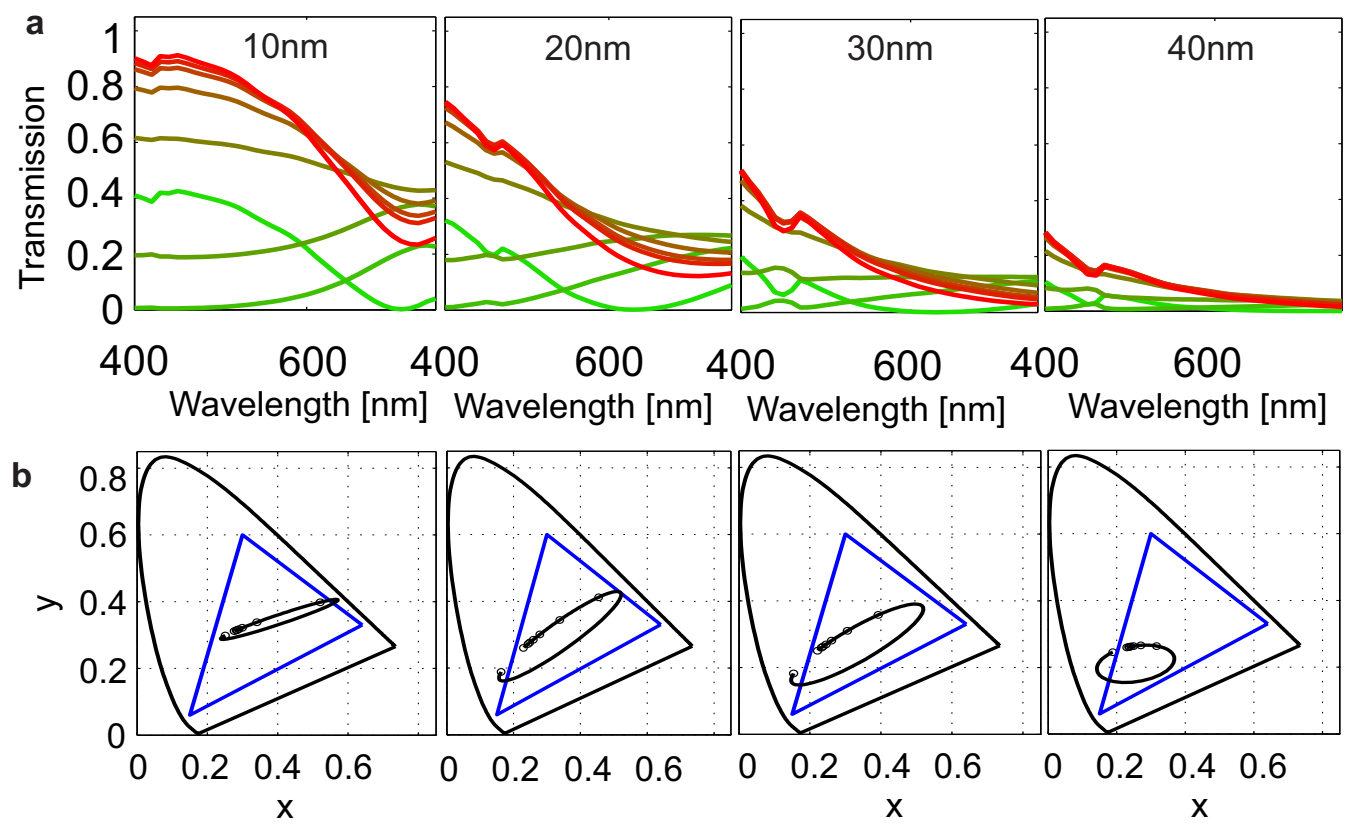

Figure 3. (a) Polarized transmittance for analyzer angles corresponding to Fig. 2 for different silver film thicknesses $t$. The period $p$ is $160 \mathrm{~nm}$ and the silver wire width $w=110 \mathrm{~nm}$. (b) Corresponding CIE color plot.
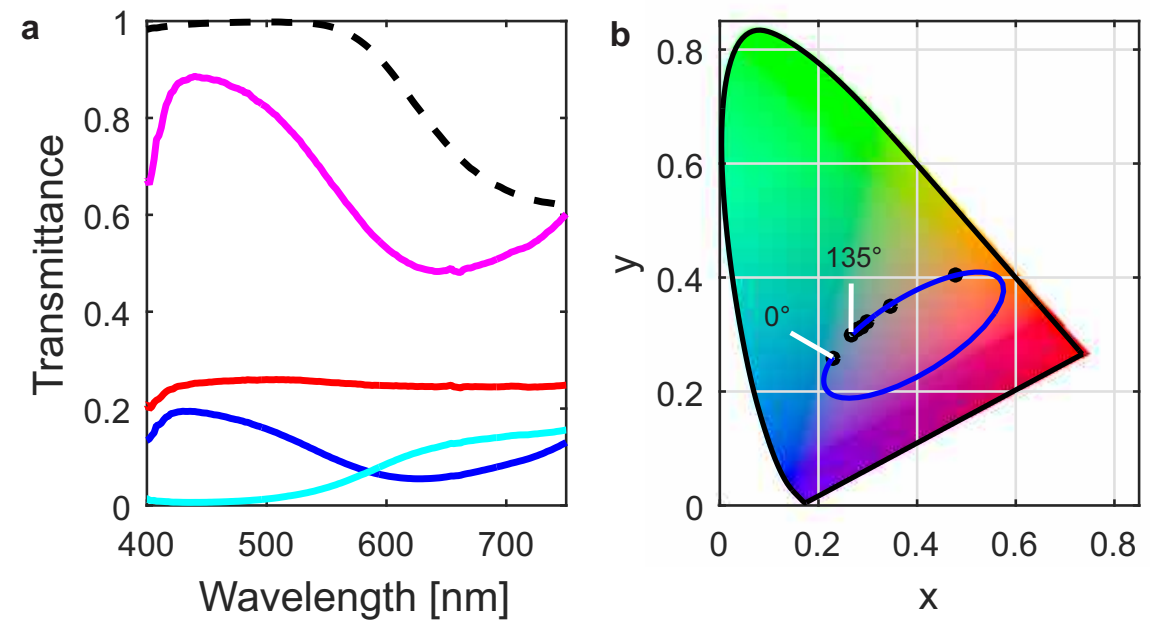

Figure 4. (a) Reconstruction of transmission spectra through the system of Fig. $1 \mathrm{~b}$ as for different analyzer orientations. Dashed line: $\cos (\Delta \phi)$ with $\Delta \phi$ measured by ellipsometry. Blue- $\theta=0$, cyan- $\theta=\pi / 4$, red $-\theta=\pi / 2$, purple- $\theta=3 \pi / 4$. (b) Corresponding CIE color coordinates.

as violet, magenta, yellow, cyan, turquoise and different tones of white are generated at intermediate voltages. Overall, an area above $70 \%$ of the color gamut of standard RGB filters is generated with a single filter. This spectral selectivity is added in transmission without any loss in the image resolution. Compared to liquid crystal tunable filters alone (Fig. 5a), the resonance spectral width is decreased from $180 \mathrm{~nm}$ to $120 \mathrm{~nm}$ in the green range. Other configurations, not shown here, can enhance the red color saturation. The presented approach is foreseen to be implemented in a variety of devices including miniature sensors or smart-phone cameras to enhance the color information, ultra-flat multispectral imagers, wearable or head-worn displays as well as high resolution display panels. 
a
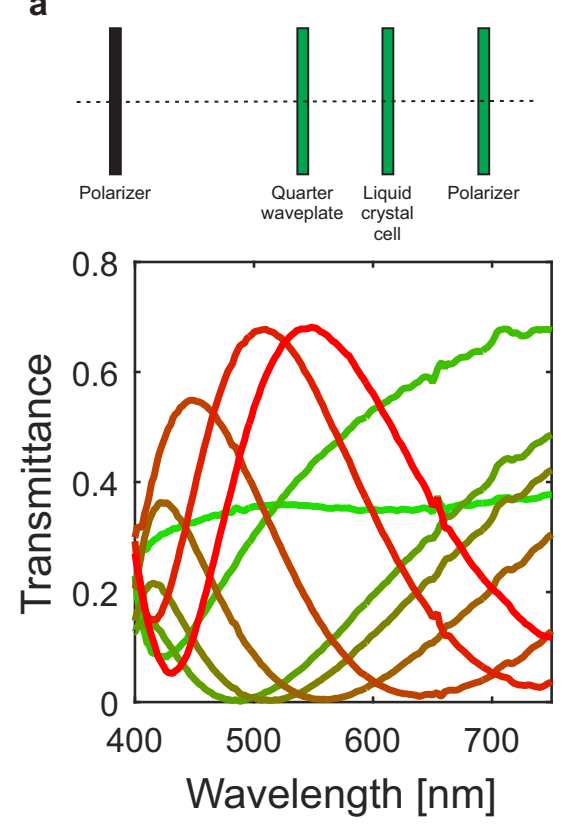
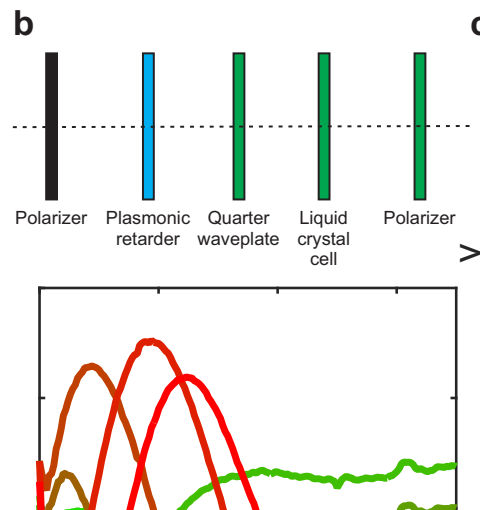

$>0$

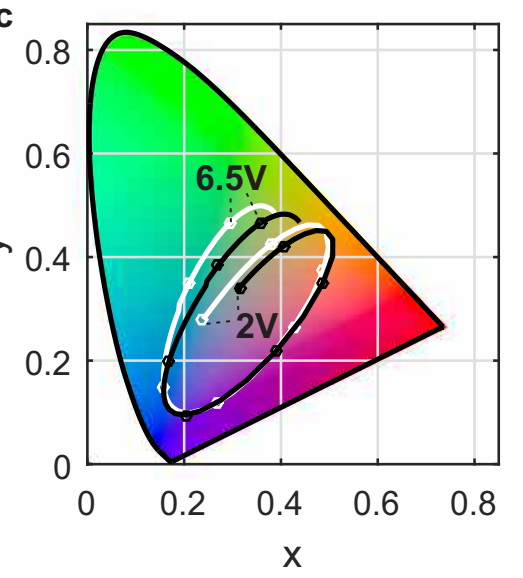

$2 \mathrm{~V} 3.15 \mathrm{~V} \quad 3.45 \mathrm{~V} \quad 3.55 \mathrm{~V} \quad 3.75 \mathrm{~V} \quad 4.2 \mathrm{~V} \quad 5.25 \mathrm{~V} \quad 6.5 \mathrm{~V}$

Figure 5. Reconstruction of color transmitted through the tunable filter. (a) Liquid crystal cell only. (b) With plasmonic nanostructure oriented at $\psi=4^{\circ}$ (configuration of Fig. 1a. (c) Corresponding CIE color plot with configurations of panel a (black) and panel b (white).

\section{ACKNOWLEDGMENTS}

Funding from Nano-Argovia is gratefully acknowledged.

\section{REFERENCES}

[1] Duempelmann, L., Luu-Dinh, A., Gallinet, B., and Novotny, L., "Four-fold color filter based on plasmonic phase retarder," ACS Photonics 3, 190-196 (2016).

[2] Duempelmann, L., Gallinet, B., and Novotny, L., "Multispectral imaging with tunable plasmonic filters," ACS Photonics 4, 236-241 (2017).

[3] Driencourt, L., Federspiel, F., Kazazis, D., Tseng, L.-T., Frantz, R., Ekinci, Y., Ferrini, R., and Gallinet, B., "Electrically tunable multicolored filter using birefringent plasmonic resonators and liquid crystals," ACS Photonics $\mathbf{0}(\mathrm{ja})$, null (0).

[4] Quaranta, G., Basset, G., Martin, O. J. F., and Gallinet, B., "Recent advances in resonant waveguide gratings," Laser Photonics Rev. 12(9), 1800017 (2018).

[5] Johnson, P. B. and Christy, R. W., "Optical-Constants of Noble-Metals," Phys. Rev. B 6, 4370 (1972).

[6] Lütolf, F., Martin, O. J. F., and Gallinet, B., "Fano-resonant aluminum and gold nanostructures created with a tunable, up-scalable process," Nanoscale 7, 18179-18187 (2015).

[7] Lütolf, F., Casari, D., and Gallinet, B., "Low-cost and large-area strain sensors based on plasmonic fano resonances," Adv. Opt. Mat. 4, 715-721 (2016). 